\title{
Antígeno prostático específico (PSA) relacionado al perfil antropométrico en pacientes del Hospital II Huamanga Carlos Tuppia García-Godos, EsSalud. Ayacucho
}

Cinthia Gavilán Zamora 1,a; Emilio Germán Ramírez Roca 1,b; Nancy Victoria Castilla-Torres¹,c

\section{RESUMEN}

Objetivo: Determinar la asociación del nivel de antígeno prostático específico (PSA) plasmático y PSA masa según riesgo de padecer enfermedades prostáticas con el perfil antropométrico.

Materiales y métodos: Estudio correlacional, de enfoque cuantitativo de dimensión transversal y retrospectiva. La muestra estuvo constituida por 156 historias clínicas de pacientes varones, con pruebas de PSA y datos antropométricos. Para el análisis de la relación de las variables se utilizó la prueba Rho de Spearman, con un nivel de confianza de $95 \%$. Resultados: La edad promedio de los pacientes fue $67,85 \pm 10,83$ años y presentaron un valor medio de PSA de $3,57 \pm 7,30 \mathrm{ng} / \mathrm{mL}$. El 9,60\% (15 pacientes) tuvo un riesgo bajo de padecer enfermedades prostáticas (PSA = 4,1-9,90 ng/mL); el 5,10\% (8 individuos) mostró riesgo intermedio ( $\mathrm{PSA}=10-19,90 \mathrm{ng} / \mathrm{mL})$; y el 3, $80 \%(6$ pacientes) tuvo un riesgo alto (PSA $\geq 20 \mathrm{ng} / \mathrm{mL})$. El promedio del índice de masa corporal (IMC) fue $26,37 \pm 3,81 \mathrm{~kg} / \mathrm{m}^{2}: 85$ pacientes (54,50\%) tenían sobrepeso; y 18 (11,50\%), obesidad. La

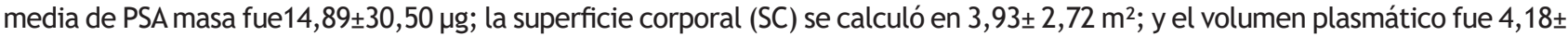
$0,21 \mathrm{~L}$. Se evidenció una correlación positiva muy baja entre el PSA plasmático y la edad (rho =0,184; $p=0,022$ ), así como con entre la PSA masa y la edad (rho $=0,176 ; p=0,028$ ). Se obtuvo una asociación positiva moderada entre el PSA plasmático y la superficie corporal (SC) (rho =0,456; $p=0,000)$; y entre el PSA masa y SC (rho =0,463; $p=0,000$ ). No se encontró relación entre el IMC y el PSA.

Conclusiones: Se evidenció la asociación entre el valor de PSA plasmático y PSA masa con el perfil antropométrico, según el riesgo de padecer enfermedades prostáticas, que fue mayor con la superficie corporal y la edad.

Palabras clave: Antígeno prostático específico (PSA); Antropometría; Índice de masa corporal (IMC) (Fuente: DeSC BIREME).

\section{Prostate-specific antigen (PSA) related to the anthropometric profile among patients admitted at the Hospital II Huamanga Carlos Tuppia García-Godos, EsSalud. Ayacucho}

\section{ABSTRACT}

Objective: To determine the association between plasma and mass prostate-specific antigen (PSA) levels and the anthropometric profile, taking into account the risk of prostate pathologies.

Materials and methods: A correlational, quantitative, cross-sectional and retrospective study conducted with a sample of 156 medical records of male patients with PSA tests and anthropometric data. Spearman's Rho with a $95 \%$ confidence level was used to analyze the relationship between the variables.

Results: The average age of the patients was $67.85 \pm 10.83$ years and their mean PSA value was $3.57 \pm 7.30 \mathrm{ng} / \mathrm{mL}$. Fifteen $(15)$ patients $(9.60 \%)$ had a low risk (PSA $=4.1-9.90 \mathrm{ng} / \mathrm{mL})$, eight $(5.10 \%)$ a medium risk (PSA $=10-19.90 \mathrm{ng} / \mathrm{mL}) \mathrm{and} \mathrm{six}(3.80 \%)$ a high risk (PSA $\geq 20 \mathrm{ng} / \mathrm{mL}$ ) of developing prostate pathologies. The mean body mass index (BMI) was $26.37 \pm 3.81 \mathrm{~kg} / \mathrm{m}^{2}: 85$ patients (54.50\%) were overweight and $18(11.50 \%)$ were obese. The mean mass PSA was $14.89 \pm 30.50 \mu \mathrm{g}$, the body surface area (BSA) was $3.93 \pm 2.72 \mathrm{~m}^{2}$ and the plasma volume was $4.18 \pm 0.21 \mathrm{~L}$. A very low positive correlation was evidenced between plasma PSA and age (rho $=0.184 ; p=0.022$ ) and between mass PSA and age (rho $=0.176 ; p=0.028$ ). There was a moderate positive association between plasma PSA and BSA ( $r$ o $=0.456 ; p=0.000$ ) and between mass PSA and BSA (rho $=0.463 ; p=0.000)$. No relationship was found between BMI and PSA.

Conclusions: The association between plasma and mass PSA levels and the anthropometric profile was demonstrated, taking into account the risk of prostate pathologies, which increased with BSA and age.

Keywords: Prostate-specific antigen (PSA); Anthropometry; Body mass index (BMI) (Source: MeSH NLM).

1 Universidad Nacional de San Cristóbal de Huamanga (UNSCH), Facultad de Ciencias de la Salud. Ayacucho, Perú.

a Químico Farmacéutica.

b Químico Farmacéutico, Doctor en Farmacia y Bioquímica.

c Químico Farmacéutica, Doctora en Salud Pública.

* Autor corresponsal. 


\section{INTRODUCCIÓN}

El examen del antígeno prostático específico (PSA) como marcador tumoral es una técnica de tamizaje en la detección temprana de enfermedades urológicas, ya que es mucho más sensible y específico si se compara con el tacto rectal y la ecografía transvesical ${ }^{(1,2)}$.

En la actualidad, las dolencias de la próstata, como la hiperplasia benigna prostática (HBP) y el cáncer de próstata (CaP), son un problema importante en todo el mundo. La incidencia de estas enfermedades se incrementa de manera progresiva en varones de edad avanzada. El cáncer de próstata es una neoplasia de órgano sólido que se diagnostica tardíamente porque no muestra signos ni síntomas en las primeras etapas. Por esta razón, la detección temprana es prioritaria, ya que brinda más oportunidades de aplicar distintas alternativas terapéuticas, lo que optimiza la calidad de vida e incrementa el tiempo de supervivencia del paciente ${ }^{(1,3-5)}$.

La Organización Mundial de Salud (OMS) informó que uno de cada siete hombres mayores de 50 años fue diagnosticado con cáncer de próstata, que es una de las principales causas de muerte en individuos mayores, y la segunda más común, después de cáncer de pulmón ${ }^{(2)}$.

Para el 2020, el Instituto Nacional del Cáncer (NIH) estimó que se alcanzaría un total de 191930 nuevos casos de cáncer de próstata y que 33330 personas morirían a causa de esta enfermedad en el mundo ${ }^{(6)}$.

En el Perú, se considera que el $60 \%$ de hombres mayores de 50 años padece de algún tipo de enfermedad prostática; de ellas, la más común es la HPB. En el 2019 se diagnosticaron 7598 casos nuevos de cáncer, lo que representó el 11,40\% de todas las neoplasias malignas; además, 2721 muertes fueron reportadas, por lo que se convirtió en primera causa de muerte por cáncer en hombres ${ }^{(4,7)}$.

El impacto del CaP en la salud pública es enorme y lo seguirá siendo en las próximas décadas. En la actualidad, existe un número elevado de diagnósticos en fases muy avanzadas de la enfermedad, lo que incrementa la mortalidad, y es la razón por la cual existen diversos estudios enfocados en la prueba de PSA ${ }^{(8)}$.

Diversas investigaciones señalan que la composición corporal influye en la concentración plasmática de antígeno prostático específico (PSA, del inglés prostate-specific antigen). Es así que los pacientes obesos reportan menores valores de PSA que los normopesos, debido a una mayor hemodilución y una menor actividad androgénica: esto ocasiona que aparezcan resultados negativos falsos de CaP, lo que dificulta el diagnóstico oportuno. La composición corporal puede expresarse en perfiles antropométricos como peso, talla, índice masa corporal (IMC) o superficie corporal (SC) ${ }^{(9-11)}$.

En el Perú no existen investigaciones que incluyan los criterios mencionados. La relevancia de este trabajo radica en que emplea la antropometría directa e indirecta para establecer el riesgo de sufrir una enfermedad prostática e identifica otros factores predisponentes relacionados a las variables señaladas. Todo ello permitirá plantear nuevas estrategias que contribuyan a la detección temprana de estos cuadros y que enfaticen la necesidad de mejorar el estilo de vida para prevenir el cáncer de próstata y reducir su morbimortalidad.

Por ello, esta investigación se enfoca en en determinar la concentración de antígeno prostático específico (PSA), de acuerdo al riesgo de padecer enfermedades prostáticas, y evaluar su relación con el perfil antropométrico del paciente.

\section{MATERIALES Y MÉTODOS}

\section{Diseño y población de estudio}

La investigación tuvo un diseño descriptivo correlacional, transversal, retrospectivo y de enfoque cuantitativo. La muestra estuvo constituida por 156 historias clínicas, seleccionadas mediante muestreo aleatorio simple (MAS), que correspondían a la población de pacientes masculinos, entre 30 a 80 años, que acudieron al Servicio de Urología del Hospital II de Huamanga Carlos Tuppia García-Godos, EsSalud Ayacucho, del 15 de marzo al 15 de setiembre de 2019. Los datos que se obtuvieron fueron los resultados de las pruebas de antígeno prostático específico (PSA) y los datos del perfil antropométrico como talla y peso.

\section{Variables y mediciones}

El PSA y el perfil antropométrico (talla y peso) fueron las variables de la investigación. Esta información se obtuvo de las historias clínicas del Servicio de Urología y de los resultados archivados en el Laboratorio del hospital.

Antígeno prostático específico (PSA) total plasmático: Este dato se obtuvo de cada una de las historias clínicas. El PSA se registró en nanogramos por mililitro $(\mathrm{ng} / \mathrm{ml})$ y los valores se clasificaron en los siguientes rangos, según riesgo de padecer enfermedades prostáticas ${ }^{(7,12)}$ :

Normal $(0-4 \mathrm{ng} / \mathrm{mL})$

Bajo riesgo $(4,1-9,90 \mathrm{ng} / \mathrm{mL})$

Riesgo intermedio $(10-19,90 \mathrm{ng} / \mathrm{mL})$

Alto riesgo $(\geq 20 \mathrm{ng} / \mathrm{mL})$

-PSA masa ( $\mu \mathrm{g})$ : Es la cantidad absoluta de proteína PSA circulante secretada por la próstata e independiente del volumen plasmático. Su valor se calculó según la siguiente fórmula ${ }^{(10,11)}$ :

PSA masa $(\mu \mathrm{g})=[\mathrm{PSA}] \times$ volumen plasmático $(\mathrm{VP})$ 
Antígeno prostático específico (PSA) relacionado al perfil

antropométrico en pacientes del Hospital II Huamanga Carlos

Tuppia García-Godos, EsSalud. Ayacucho

-Perfil antropométrico: expresado en antropometría directa e indirecta.

Antropometría directa ${ }^{(13)}$ :

Edad (años): Se categorizaron del siguiente modo:

Adulto joven (20-39 años)

Adulto medio (40-49 años)

Adulto maduro (50-59 años)

Adulto mayor $\geq 60$ años

Peso (kg): Provee una evaluación a grandes rasgos de toda la composición corporal, dato que se obtuvo de las historias clínicas.

Talla $(m)$ : Es la medida para determinar el peso ideal, obtenida de las historias clínicas.

Antropometría indirecta: Parámetros calculados con las siguientes fórmulas ${ }^{(10,11,13)}$ :

Índice de masa corporal (IMC): Es un indicador de la densidad corporal que explica las diferencias en la composición del cuerpo al definir el nivel de adiposidad, con base en la relación entre peso corporal en kilogramos (kg) y talla en metros cuadrados $\left(\mathrm{m}^{2}\right)$, que evidencia el estado nutricional. Según el IMC los pacientes fueron clasificados en las siguientes categorías:

Insuficiencia ponderal $\left(<18,50 \mathrm{~kg} / \mathrm{m}^{2}\right)$

Normal $\left(18,50-24,90 \mathrm{~kg} / \mathrm{m}^{2}\right)$

Sobrepeso $\left(25,00-29,9 \mathrm{~kg} / \mathrm{m}^{2}\right)$

Obesidad $\left(\geq 30 \mathrm{~kg} / \mathrm{m}^{2}\right)$

Superficie corporal $(\mathrm{SC})\left(\mathrm{m}^{2}\right)$ : se obtuvo con la siguiente fórmula:

(SC): (Peso kg)0,425 x (talla cm)0,72 x 0,007184

Volumen plasmático (VP) (litros): se obtuvo con la siguiente fórmula:

VP: SC x 1,670.

\section{Análisis estadístico}

Los datos fueron procesados, ordenados y codificados en una hoja de cálculo Microsoft Office Excel, y luego se empleó el paquete estadístico SPSS (Statistical Package for Social Sciences) para Windows.

Se calcularon los estadísticos descriptivos como media aritmética $(\bar{x})$ y desviación estándar (DE). El coeficiente de correlación de Spearman, previa evaluación de la distribución normal con el test de Kolmogorov-Smirnov, se aplicó para la estadística inferencial (establecer las posibles diferencias y asociaciones entre las variables del perfil antropométrico y el PSA). En todos los casos se estableció un nivel de confianza del $95 \%$.

\section{Consideraciones éticas}

Dado que la investigación fue producto del análisis de datos secundarios de acceso público, luego de obtener el permiso correspondiente de la dirección del hospital, la información se codificó y se conservó en el anonimato, de acuerdo con la declaración de Helsinski.

\section{RESULTADOS}

De 156 de pacientes que intervinieron en el estudio, 125 $(80,10 \%)$ fueron adultos mayores, con una edad promedio de $67,85 \pm 10,83$ años y una media de $3,57 \pm 7,30 \mathrm{ng} / \mathrm{mL}$ de PSA. Destacamos que 15 pacientes $(9,60 \%)$ presentaron valores de bajo riesgo de padecer enfermedades prostáticas. El peso medio fue $69,47 \pm 10,91 \mathrm{~kg}$ y la talla media era $1,62 \pm 0,07 \mathrm{~m}$. El promedio de IMC fue $26,37 \pm 3,81 \mathrm{~kg} / \mathrm{m}^{2}$ en promedio $y$, según este parámetro, 85 pacientes $(54,50 \%)$ tenían sobrepeso. El PSA masa mostró un valor de 14,89 $\pm 30,50 \mu \mathrm{g}$; la superficie corporal midió $3,93 \pm 2,72 \mathrm{~m}^{2}$; y el volumen plasmático fue $4,18 \pm 0,21 \mathrm{~L}$ (Tabla 1 ).

Tabla 1. Niveles de antígeno prostático específico (PSA) y perfil antropométrico de pacientes del Hospital II Huamanga Carlos Tuppia García-Godos, EsSalud. Ayacucho

\begin{tabular}{|c|c|c|c|c|c|}
\hline \multirow[b]{2}{*}{ Parámetros } & \multicolumn{4}{|c|}{ Rangos de edad } & \multirow[b]{2}{*}{ Total } \\
\hline & $\begin{array}{l}\text { Adulto joven } \\
\text { (20-39 años) }\end{array}$ & $\begin{array}{l}\text { Adulto medio } \\
\text { (40 - } 49 \text { años) }\end{array}$ & $\begin{array}{r}\text { Adulto maduro } \\
\text { (50 - } 59 \text { años) }\end{array}$ & $\begin{array}{l}\text { Adulto mayor } \\
\text { ( } \geq 60 \text { años) }\end{array}$ & \\
\hline \multicolumn{6}{|l|}{ Valores de antígeno prostático específico (PSA) } \\
\hline \multicolumn{6}{|l|}{ Niveles de PSA (ng/mL) } \\
\hline Normal (0-4), n (\%) & $2(1,30)$ & $7(4,50)$ & $22(14,10)$ & $96(61,50)$ & $127(81,40)$ \\
\hline Bajo riesgo $(4,10-9,90), \mathrm{n}(\%)$ & $0(0,00)$ & $0(0,00)$ & $0(0,00)$ & $15(9,60)$ & $15(9,60)$ \\
\hline Riesgo intermedio $(10-19,90), n(\%)$ & $0(0,00)$ & $0(0,00)$ & $0(0,00)$ & $8(5,10)$ & $8(5,10)$ \\
\hline Alto riesgo $(\geq 20), n^{\circ}(\%)$ & $0(0,00)$ & $0(0,00)$ & $0(0,00)$ & $6(3,80)$ & $6(3,80)$ \\
\hline Total, n (\%) & $2(1,30)$ & $7(4,50)$ & $22(14,10)$ & $125(80,10)$ & $156(100,00)$ \\
\hline PSA (ng/mL), $(\bar{x} \pm D E)$ & $0,58 \pm 0,58$ & $0,79 \pm 0,89$ & $1,45 \pm 1,11$ & $4,15 \pm 8,04$ & $3,57 \pm 7,30$ \\
\hline PSA (masa) $(\mu \mathrm{g}),(\bar{x} \pm D E)$ & $2,6 \pm 2,54$ & $3,45 \pm 3,89$ & $6,09 \pm 4,64$ & $17,28 \pm 33,61$ & $14,89 \pm 30,50$ \\
\hline
\end{tabular}




\begin{tabular}{|c|c|c|c|c|c|}
\hline \multirow[b]{2}{*}{ Parámetros } & \multicolumn{4}{|c|}{ Rangos de edad } & \multirow[b]{2}{*}{ Total } \\
\hline & $\begin{array}{l}\text { Adulto joven } \\
\text { (20-39 años) }\end{array}$ & $\begin{array}{l}\text { Adulto medio } \\
\text { (40 - } 49 \text { años) }\end{array}$ & $\begin{array}{r}\text { Adulto maduro } \\
\text { (50 - } 59 \text { años) }\end{array}$ & $\begin{array}{l}\text { Adulto mayor } \\
\text { ( } \geq 60 \text { años) }\end{array}$ & \\
\hline \multicolumn{6}{|l|}{ Valores de perfil antropométrico } \\
\hline \multicolumn{6}{|l|}{ Rangos de índice de masa corporal kg/m² } \\
\hline Insuficiencia ponderal $\left(<18,5 \mathrm{~kg} / \mathrm{m}^{2}\right), \mathrm{n}(\%)$ & $0(0,00)$ & $1(0,60)$ & $0(0,00)$ & $1(0,60)$ & $2(1,30)$ \\
\hline Normal $(18,5-24,9$ kg/m²), n (\%) & $1(0,60)$ & $1(0,60)$ & $5(3,20)$ & $44(28,20)$ & $51(32,70)$ \\
\hline Sobrepeso $\left(25,0-29,9 \mathrm{~kg} / \mathrm{m}^{2}\right), \mathrm{n}(\%)$ & $0(0,00)$ & $5(3,20)$ & $12(07,70)$ & $68(43,60)$ & $85(54,50)$ \\
\hline Obesidad ( $\geq 30$ kg $\left./ \mathrm{m}^{2}\right), \mathrm{n}(\%)$ & $1(0,60)$ & $0(0,00)$ & $5(3,20)$ & $12(7,70)$ & $18(11,50)$ \\
\hline Total, n (\%) & $2(1,30)$ & $7(4,50)$ & $22(14,10)$ & $125(80,10)$ & $156(100,00)$ \\
\hline Edad (años), ( $\bar{x} \pm \mathrm{DE})$ & $34,50 \pm 6,36$ & $45,71 \pm 2,56$ & $53,86 \pm 2,4$ & $70,09 \pm 6,83$ & $67,85 \pm 10,83$ \\
\hline Peso (kg), ( $\bar{x} \pm \mathrm{DE})$ & $81 \pm 5,66$ & $70,71 \pm 11,16$ & $73,0 \pm 14,40 \quad 6$ & $68,59 \pm 10,55$ & $69,47 \pm 10,91$ \\
\hline Talla (m), ( $\bar{x} \pm \mathrm{DE})$ & $1,66 \pm 0,14$ & $1,68 \pm 0,037$ & $1,63 \pm 0,07$ & $1,61 \pm 0,07$ & $1,62 \pm 0,07$ \\
\hline IMC $\left(\mathrm{kg} / \mathrm{m}^{2}\right),(\overline{\mathrm{x}} \pm \mathrm{DE})$ & $29,89 \pm 7,12$ & $25,02 \pm 4,05$ & $27,41 \pm 3,72$ & $26,21 \pm 3,46$ & $26,37 \pm 3,81$ \\
\hline$S C\left(m^{2}\right),(\bar{x} \pm D E$ & $5,07 \pm 5,20$ & $3,03 \pm 2,71$ & $4,38 \pm 2,72$ & $3,89 \pm 2,7$ & $3,93 \pm 2,72$ \\
\hline VP (L), ( $\bar{x} \pm D E)$ & $4,29 \pm 0,42$ & $4,35 \pm 0,11$ & $4,19 \pm 0,21$ & $4,16 \pm 0,21$ & $4,18 \pm 0,21$ \\
\hline
\end{tabular}

$\overline{\mathrm{x}}:$ Media

$\mathrm{DE}$ : desviación estándar

IMC: Índice de masa corporal

SC: Superficie corporal

VP: Volumen plasmático

Existe una relación positiva débil significativa entre la concentración de antígeno prostático específico (PSA) y la edad (rho $=0,184 ; p=0,022$ ). El grupo de pacientes adultos mayores de 60 años presentó bajo riesgo de padecer enfermedades prostáticas, a diferencia de los grupos adulto joven, adulto medio y adulto maduro, que no mostraron ningún riesgo (Tabla 2 ).

Tabla 2. Relación de la concentración de antígeno prostático específico (PSA) según riesgo de padecer enfermedades prostáticas y la edad en pacientes del Hospital II Huamanga Carlos Tuppia García-Godos, EsSalud. Ayacucho

\begin{tabular}{|c|c|c|c|c|c|c|c|}
\hline \multirow[b]{2}{*}{ Rangos de edad (años) } & \multicolumn{5}{|c|}{$\begin{array}{l}\text { Rangos de antígeno prostático especifico según riesgo de } \\
\text { padecer enfermedades prostáticas ( } \mathrm{ng} / \mathrm{mL})\end{array}$} & \multirow[b]{2}{*}{$\begin{array}{l}\text { *Rho de } \\
\text { Sperman }\end{array}$} & \multirow[b]{2}{*}{$\begin{array}{l}\text { **Valo } \\
\text { de } p\end{array}$} \\
\hline & $\begin{array}{c}\text { Sin riesgo } \\
(0-4)\end{array}$ & $\begin{array}{l}\text { Bajo riesgo } \\
(4,10-9,90)\end{array}$ & $\begin{array}{c}\text { Riesgo } \\
\text { intermedio } \\
(10-19,90)\end{array}$ & $\begin{array}{l}\text { Alto riesgo } \\
(\geq 20)\end{array}$ & Total & & \\
\hline Adulto joven (20-39), n (\%) & $2(1,30)$ & $0(0,00)$ & $0(0,00)$ & $0(0,00)$ & $2(1,30)$ & 0,184 & 0,022 \\
\hline Adulto medio (40-49), n (\%) & $7(4,50)$ & $0(0,00)$ & $0(0,00)$ & $0(0,00)$ & $7(4,50)$ & & \\
\hline Adulto maduro (50-59), n (\%) & $22(14,10)$ & $0(0,00)$ & $0(0,00)$ & $0(0,00)$ & $22(14,10)$ & & \\
\hline Adulto mayor $\geq 60, \mathrm{n}(\%)$ & $96(61,50)$ & $15(9,60)$ & $8(5,10)$ & $6(3,80)$ & $125(80,10)$ & & \\
\hline Total, n (\%) & $127(81,40)$ & $15(9,60)$ & $8(5,10)$ & $6(3,80)$ & $156(100,00)$ & & \\
\hline Media de edad (años) $(\bar{x} \pm D E)$ & $66,41 \pm 11,18$ & $74,93 \pm 6,72$ & $63 \pm 5,57 \quad 74$ & $4,17 \pm 5,56$ & $68,59 \pm 10,55$ & & \\
\hline
\end{tabular}

*Correlación de Spearman

**Valor de $p \leq 0,05$

$\overline{\mathrm{X}}$ : Media

DE: desviación estándar 
Antígeno prostático específico (PSA) relacionado al perfil antropométrico en pacientes del Hospital II Huamanga Carlos Tuppia García-Godos, EsSalud. Ayacucho

Encontramos una correlación positiva baja entre el PSA masa y la edad ( $r h o=0,176 ; p=0,028$ ) (Figura 1).

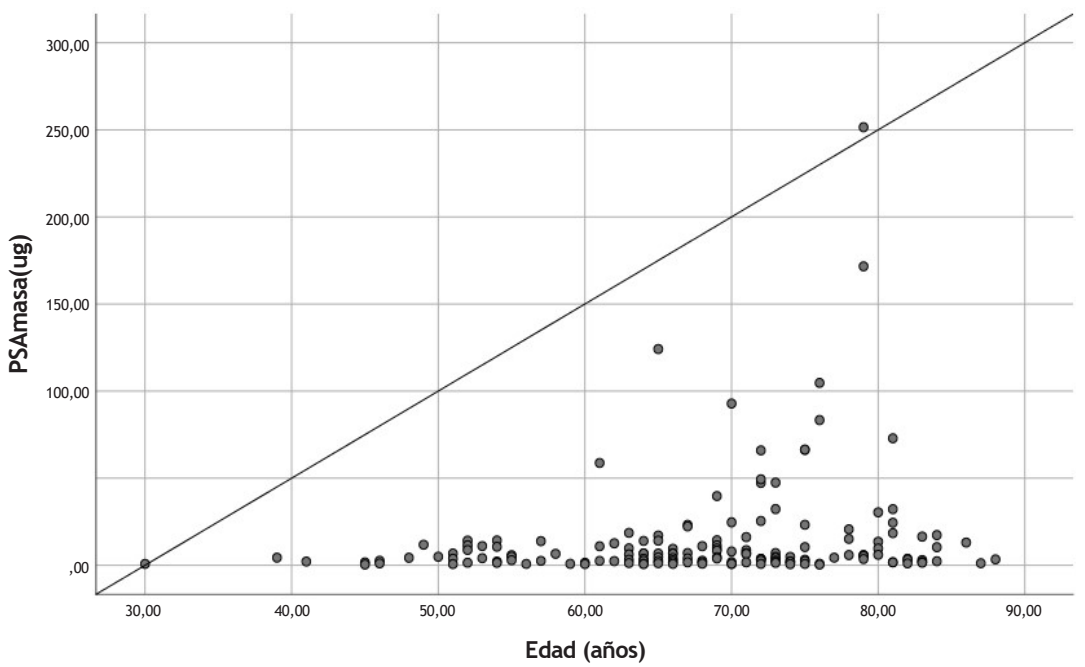

rho $=0,176 ; p=0,028$

Figura 1. Relación entre PSA masa y edad

Además, se identificó una asociación positiva moderada significativa entre el PSA y la superficie corporal (SC) (rho $=0,456$; $p=0,00$ ). Sin embargo, no se encontró correlación entre la PSA, IMC, peso, talla ni volumen plasmático (Tabla 3 ).

Tabla 3. Relación de la concentración de antígeno prostático específico (PSA) según riesgo de padecer enfermedades prostáticas y el perfil antropométrico en pacientes del Hospital II Huamanga Carlos Tuppia García-Godos, EsSalud. Ayacucho

\begin{tabular}{|c|c|c|c|c|c|c|c|}
\hline \multirow[b]{2}{*}{ Rangos de edad (años) } & \multicolumn{5}{|c|}{$\begin{array}{l}\text { Rangos de antígeno prostático específico según riesgo de } \\
\text { padecer enfermedades prostáticas (ng/mL) }\end{array}$} & \multirow[b]{2}{*}{$\begin{array}{l}\text { *Rho de } \\
\text { Sperman }\end{array}$} & \multirow[b]{2}{*}{$\begin{array}{l}* * \text { Valor } \\
\text { de } p\end{array}$} \\
\hline & $\begin{array}{l}\text { Sin riesgo } \\
(0-4)\end{array}$ & $\begin{array}{l}\text { Bajo riesgo } \\
(4,10-9,90)\end{array}$ & $\begin{array}{c}\text { Riesgo } \\
\text { intermedio } \\
(10-19,90)\end{array}$ & $\begin{array}{l}\text { Alto riesgo } \\
0 \quad(\geq 20)\end{array}$ & Total & & \\
\hline \multicolumn{8}{|l|}{ IMC } \\
\hline Insuficiencia ponderal $(<18,5), \mathrm{n}(\%)$ & $2(1,30)$ & $0(0,00)$ & $0(0,00)$ & $0(0,00)$ & $2(1,30)$ & 0,029 & 0,721 \\
\hline Normal $(18,5-24,9), n$ (\%) & $38(24,40)$ & $4(2,60)$ & $4(2,60)$ & $5(3,20)$ & $51(32,70)$ & & \\
\hline Sobrepeso $(25,0-29,9), n(\%)$ & $74(47,40)$ & $7(4,50)$ & $3(1,90)$ & $1(0,60)$ & $85(54,50)$ & & \\
\hline Obesidad ( $\geq 30$ ), n (\%) & $13(8,30)$ & $4(2,60)$ & $1(0,60)$ & $0(0,00)$ & $18(11,50)$ & & \\
\hline Total, n (\%) & $127(81,40)$ & $15(9,60)$ & $8(5,10)$ & $6(3,80)$ & $156(100,00)$ & & \\
\hline Media de IMC $\left(\mathrm{kg} / \mathrm{m}^{2}\right),(\overline{\mathrm{x}} \pm \mathrm{DE})$ & $26,56 \pm 3,33$ & $28,01 \pm 5,07$ & $26,09 \pm 3,06 \quad 22$ & $22,98 \pm 3,11$ & $3,89 \pm 2,70$ & & \\
\hline Peso (kg), $(\bar{x} \pm D E)$ & $69,53 \pm 10,19$ & $73,67 \pm 14,88$ & $69,13 \pm 12,045 \varepsilon$ & $58,33 \pm 6,95$ & $1,61 \pm 0,07$ & 0,024 & 0,763 \\
\hline Talla $(m),(\bar{x} \pm D E)$ & $1,63 \pm 0,07$ & $1,63 \pm 0,09$ & $1,62 \pm 0,08$ & $1,59 \pm 0,11$ & $26,21 \pm 3,46$ & 0,073 & 0,367 \\
\hline$S C\left(m^{2}\right),(\bar{x} \pm D E)$ & $4,26 \pm 2,92$ & $3,58 \pm 0,16$ & $2,51 \pm 0,14$ & $2,46 \pm 0,19$ & $4,16 \pm 0,21$ & 0,456 & 0,000 \\
\hline VP (L), ( $\bar{x} \pm \mathrm{DE})$ & $4,18 \pm 0,20$ & $4,18 \pm 0,27$ & $4,19 \pm 0,24$ & $4,10 \pm 0,32$ & $68,59 \pm 10,55$ & 0,073 & 0,638 \\
\hline
\end{tabular}

*Correlación de Spearman

**Valor de $p \leq 0,05$

$\overline{\mathrm{X}}$ : Media

DE: desviación estándar

IMC: Índice de masa corporal

SC: Superficie corporal

VP: Volumen plasmático 


\section{DISCUSIÓN}

La investigación en 165 pacientes reportó una media de antígeno prostático específico (PSA) de $3,57 \pm 7,30 \mathrm{ng} / \mathrm{mL}$ y cantidades absolutas circulantes (expresadas como PSA masa) de 14,89 $\pm 30,50 \mu \mathrm{g}$; este valor fue calculado a partir de la concentración plasmática de PSA y el volumen plasmático (VP). En relación al riesgo de tener una enfermedad prostática, este estudio mostró que en el grupo de adultos mayores ( $\geq 60$ años), el 9,60\% (15 pacientes) estaba en bajo riesgo; el 5,10\% (8 individuos), en riesgo intermedio; y el 3,80\% (6 pacientes), en alto riesgo. Estos hallazgos son semejantes a otros trabajos, ya que este grupo de edad suele ser más vulnerable ${ }^{(14-16)}$.

El PSA es una glicoproteína con actividad enzimática producida por las células epiteliales de los túbulos y acinos prostáticos. En condiciones patológicas los niveles de PSA en la sangre se incrementan, por lo que es un biomarcador tumoral muy útil del cáncer prostático, pero no muy específico ${ }^{(5,11)}$. Existen diversas enfermedades en las que PSA está elevado, entre ellas, las afecciones benignas (no cancerosas) como la prostatitis, la hiperplasia benigna de la próstata (HBP) y la obstrucción urinaria ${ }^{(2-5,12,14)}$. Además, otros factores, como la edad, peso, talla, el peso prostático, el ejercicio físico, el estado posterior a la eyaculación, la retención urinaria, el sondaje vesical o la instrumentación de la uretra, masaje prostático, endoscopías y biopsias, podrían llevar a un falso diagnóstico, a un sobretratamiento, y a terapias y biopsias innecesarias ${ }^{(5,11)}$.

Un PSA de hasta $4 \mathrm{ng} / \mathrm{ml}$ está considerado como un valor normal en un límite superior, y las cantidades más elevadas corresponden a un mayor riesgo de $\mathrm{CaP}$, aunque un valor menor no garantiza la ausencia de la neoplasia. Los valores entre 4 y $10 \mathrm{ng} / \mathrm{ml}$ se clasifican como ligeramente elevados, de 10 a $19,90 \mathrm{ng} / \mathrm{ml}$ son moderadamente elevados, y una cantidad mayor que $20 \mathrm{ng} / \mathrm{ml}$ se considera como sumamente elevada. Es por ello que distintos autores recomiendan que los pacientes con resultados de PSA mayores que $10 \mathrm{ng} / \mathrm{ml}$ se realicen una biopsia guiada por ecografía, ya que tienen 145,30 veces más probabilidad de padecer $\mathrm{CaP}$ en los siguientes 10 años ${ }^{(17)}$. Por otro lado, a pesar de que las concentraciones que oscilan entre 2,50 a $4 \mathrm{ng} / \mathrm{ml}$ se consideran dentro de un rango normal, se debe tener en cuenta que existe el $27 \%$ de probabilidades de detectar el CaP mediante biopsia ${ }^{(5,18)}$.

Encontramos una relación positiva baja entre la concentración de PSA plasmático y PSA masa con la edad. Debido a que la media de edad de nuestros pacientes fue $67,85 \pm 0,83$ años, este hallazgo indica que, a mayor edad, hay un valor mayor de PSA y, por lo tanto, un riego mayor de padecer enfermedades prostáticas; a diferencia del grupo de adultos jóvenes, medio y maduros que no presentó ningún riesgo.
Existe evidencia de que el aumento del tamaño de la glándula prostática y los niveles de PSA se incrementan con la edad ${ }^{(5)}$, por lo que la incidencia de CaP se incrementa entre los 55 y 64 años ( $32,90 \%$ ) y entre los 65 y 74 años $(37,60 \%)^{(6)}$. Del mismo modo, el envejecimiento repercute en la reducción de la producción de testosterona sérica; sin embargo, al mantener los niveles intraprostáticos de andrógenos (por su intensificada capacidad de adaptación), la tasa de mutación y la evolución maligna de las células prostáticas se incrementarían (19).

Por otro lado, los pacientes mayores de 50 años con adenoma de próstata y un PSA mayor que $1,50 \mathrm{ng} / \mathrm{ml}$ tienen ocho veces más riesgo de manifestar retención urinaria, lo que requiere tratamiento. Los mayores de 65 años con PSA menor a $1 \mathrm{ng} / \mathrm{ml}$ tienen menor riesgo de padecer $\mathrm{CaP}$ en los siguientes 10 años ${ }^{(20)}$. La Sociedad Americana de Urología recomienda que cada año hombres mayores de 50 años acudan al médico para examinar próstata mediante un tacto rectal (TR) y analizar el PSA; mientras que los hombres con antecedentes familiares de CaP deben hacerlo a partir de los 40 años ${ }^{(17)}$.

En este estudio no hallamos una relación entre la concentración de PSA y el índice de masa corporal (IMC), aunque la mayoría de los pacientes tenían sobrepeso $(54,50 \%$ y una media de IMC $26,37 \pm 3,81 \mathrm{~kg} / \mathrm{m}^{2}$. Sin embargo, sí se encontró una asociación positiva moderada entre el PSA total y la superficie corporal (SC). Estos resultados son similares a otros trabajos que sugieren que el PSA se reduce conforme se incrementa el IMC ${ }^{(9,11)}$, ya que los pacientes obesos presentan un mayor volumen plasmático (VP) y, por lo tanto, hemodilución del PSA, lo que retrasa la detección temprana de CaP (5,9-11).

La obesidad (IMC mayor a $30 \mathrm{~kg} / \mathrm{m}^{2}$ ) interfiere con una exploración física adecuada porque el exceso de tejido adiposo dificulta la detección del tumor; esto reduce la utilidad y confiabilidad de este método complementario y retrasa la indicación de una biopsia ${ }^{(5,9-11)}$. Por ello, varios estudios afirman que existe una asociación directa entre el IMC, el tamaño y la agresividad del tumor en $\mathrm{CaP}{ }^{(8,20)}$. La obesidad es un factor de riesgo que participa en el CaP durante la etapa inicial y en el progreso a un cuadro más severo debido a una alteración de los niveles de hormonas sexuales y el incremento de metabolismo de adipocinas: de ellas, la leptina tiene efectos mitogénicos y una mayor expresión de factores de crecimiento, que contribuyen a la progresión del $\mathrm{CaP}{ }^{(9,11)}$.

A pesar de la polémica que se plantea por realizar o no la prueba de PSA para la detección precoz del CaP, se estima que este procedimiento podría resultar en un descenso de la mortalidad por esta enfermedad de entre el $20 \%$ y el $30 \%$. Sin embargo, entre un $20 \%$ y un $40 \%$ de los casos de CaP identificados podrían estar sobrediagnosticados ${ }^{(2,18)}$. 
Antígeno prostático específico (PSA) relacionado al perfil antropométrico en pacientes del Hospital II Huamanga Carlos

Tuppia García-Godos, EsSalud. Ayacucho

Dado que el cáncer de próstata $(\mathrm{CaP})$ es una de las principales causas de muerte en hombres en todo el mundo, el tamizaje de PSA es de gran importancia. A pesar de que tiene un escaso valor predictivo y que existen técnicas más sensibles y específicas, todavía es el más utilizado y extensamente aceptado ${ }^{(9,11)}$. Los resultados de esta prueba se deben contrastar con con otros estudios clínicos y patológicos a fin de que el diagnóstico, la estadificación clínica, la elección de biopsia y el pronóstico sean los correctos ${ }^{(2-5,12,14)}$.

La principal limitación de este estudio es su naturaleza retrospectiva y transversal. Personal ajeno a la investigación recolectó la información a partir de las historias clínicas que estaban en el archivo del hospital. El equipo investigador no participó en el control de calidad de las técnicas de obtención de las muestras para medir los niveles del PSA y las medidas antropométricas. Por ello, para reducir cualquier sesgo, la selección de los expedientes clínicos, la recolección de los datos y los cálculos de PSA masa y de la antropometría indirecta se realizaron cuidadosamente y de acuerdo a criterios estandarizados por diversos autores $(10,11,13)$. En consecuencia, estos resultados deben extrapolarse con cautela, considerando las características de la metodología de investigación.

Concluimos que elementos del perfil antropométrico, como la superficie corporal y la edad, se asocian de manera significativa con los valores de PSA plasmática y PSA masa, lo que indica que existe riesgo para contraer enfermedades prostáticas.

Con base en lo planteado, es necesario elegir la mejor estrategia en cuanto a las técnicas de diagnóstico y tratamiento de las enfermedades urológicas, sobre todo del cáncer de próstata, para reducir la morbimortalidad y el incalculable el gasto económico que genera esta enfermedad en los sistemas de salud de todo el mundo. Debido a su historia natural, a menudo esta neoplasia es prolongada e implica un seguimiento a largo plazo y tratamientos quirúrgicos y farmacológicos costosos, como quimioterapia y radioterapia, que tienen impacto económico en la familia, la sociedad y la salud pública $(2,8,18)$.

Finalmente, recomendamos seguir investigando exhaustivamente y realizar trabajos similares que contribuyan a mejorar el estilo de vida de los pacientes, y hallar otros biomarcadores que permitan identificar, de manera precoz, las enfermedades prostáticas y sus factores de riesgo.

Agradecimientos: Al personal del Hospital II Huamanga Carlos Tuppia García-Godos, EsSalud Ayacucho, en especial a la Unidad de Admisión y del Servicio de Laboratorio.
Contribuciones de autoría: CGZ y EGRR participaron en la concepción y ejecución del tema de investigación. NVCT contribuyó en el diseño del artículo y en la revisión del contenido. CGZ ha participado en la recolección y procesamiento de las variables. CGZ y NVCT se encargaron del análisis estadístico. Todos los autores participaron en la interpretación de los datos, en la redacción y revisión crítica del manuscrito, aprobaron la versión final y se hacen responsables del contenido.

Fuentes de financiamiento: El artículo ha sido financiado por los autores.

Conflictos de interés: Los autores declaran no tener conflictos de interés.

\section{REFERENCIAS BIBLIOGRÁFICAS}

1. Armstrong BK, Barry MJ, Frydenberg M, Gardiner RA, Haines I, Carter SM. PSA testing for men at average risk of prostate cancer. Public Health Res Pract. 2017; 27(3): e2731721-6.

2. Organización Panamericana de la Salud. Expertos regionales discuten enfoques para el tamizaje y detección temprana del cáncer de próstata en las Américas [Internet]. OPS; 2017. Disponible en: https://www.paho.org/hq/index.php?option=com_content \&view=article\&tid=13818: regional-experts-discuss-approachesfor-prostate-cancer-screening-and-early-detection-in-theamericas\&ltemid $=42459$ \&lang=es

3. Heidenreich A, Bolla M, Mason MD, Matveev V, Mottet N, Schmid HP, et al. Guía clínica sobre el cáncer de próstata [Internet]. Vol 1. European Association of Urology; 2010. 196 p. Disponible en: https: // www.aeu.es/UserFiles/01-GUIA_CLINICA_SOBRE_EL_CANCER_DE_ PROSTATA.pdf

4. Cookson MS, Lowrance W. Guía 2020 del Cáncer de Próstata Avanzado. American Urological Association [Internet]. 2020; 13: 12. Disponible en: https://caunet.org/wp-content/uploads/2020/04/ AUAEspanol0520v4.pdf

5. Robles Fernández I. Identificación de biomarcadores predictivos, pronósticos y de respuesta al tratamiento en cáncer de próstata [Tesis de posgrado]. Granada: Universidad de Granada. Departamento de Medicina Legal, Toxicología y Antropología Física; 2018.

6. National Cancer Institute. Cáncer de próstata: datos estadísticos sobre el cáncer [Internet]. NIH: USA; 2021. Disponible en: https:// seer.cancer.gov/statfacts/html/prost.html

7. Instituto Nacional de Enfermedades Neoplásicas. Cáncer de próstata es la neoplasia maligna más frecuente en varones [Internet]. INEN: Lima; 2019. Disponible en: https://portal.inen.sld.pe/cancer-deprostata-es-la-neoplasia-maligna-mas-frecuente-en-varones/

8. Navarro Cutiño M, Godoy Durán S, Contreras Duverger DM. Utilidad del antígeno prostático específico en la hiperplasia prostática benigna para la detección del cáncer. Arch Hosp Univ «Gen Calixto García». 2018; 6: 50-6.

9. Allott EH, Hursting SD. Obesity and cancer: mechanistic insights from transdisciplinary studies. Endocr Relat Cancer. 2015; 22(6): R365-86.

10. López Fontana C, Maselli ME, Pérez Elizalde R, Di Milta N, Corica Alberto P, López Laur JD. La obesidad modifica el antígeno prostático específico en hombres mayores de 45 años de edad. Arch Esp Urol. 2011; 64(1): 35-42.

11. Messina D, Soto C, Corte C, Pérez Elizalde R, López Laur JD, López 
Fontana C. Relación entre índice de masa corporal y antígeno prostático específico en varones libres de enfermedad prostática. Actual Nutr. 2013; 14(3): 228-35.

12. Instituto Nacional del Cáncer. Análisis del antígeno prostático específico (PSA). [Internet]. INC: USA; 2017. Disponible en: https:// www.cancer.gov/espanol/tipos/prostata/hoja-informativa-psa

13. Instituto Nacional de Estadística e Informática. INEI - Perú: Encuesta Demográfica y de Salud Familiar 2018 - Nacional y Regional [Internet]. INEI: Lima; 2018. Disponible en: https://www.inei.gob.pe/media/ MenuRecursivo/publicaciones_digitales/Est/Lib1656/index1.html

14. Acosta N, Varela R, Mesa JA, Serrano López ML, Cómbita AL, Sanabria-Salas MC. Biomarcadores de pronóstico en pacientes con cáncer de próstata localizado. Rev Colomb Cancerol. 2017; 21(2): 113-25.

15. Godoy Durán S, Navarro Cutiño M, Contreras Duverger DM. Antígeno Prostático Específico para la detección del cáncer en la Hiperplasia Prostática Benigna. Rev Cub Urol. 2018; 7: e43.

16. Valiente Morejón W, Junco Sena B, Padrón Vega Y, Ramos Águila YC, Castillo García I. Antígeno prostático específico como predictor del diagnóstico de adenocarcinoma prostático. Rev Finlay. 2015; 5(4): 221-7.

17. Ballentine Carter H. American Urological Association (AUA) guideline on prostate cancer detection: process and rationale. BJU Int. 2013; 112(5): 543-7.

18. Eapen RS, Herlemann A, Washington SL, Cooperberg MR. Impact of the United States Preventive Services Task Force «D» recommendation on prostate cancer screening and staging. Curr Opin Urol. 2017; 27(3): 205-9.

19. Wang K, Chen X, Bird VY, Gerke TA, Manini TM, Prosperi M. Association between Age-Related Reductions in Testosterone and Risk of Prostate Cancer - An Analysis of Patients' Data with Prostatic Diseases. Int J Cancer. 2017; 141(9): 1783-93.

20. López Ríos L, Sánchez Montero IR, García Moreno I. Relación entre el marcador tumoral antígeno prostático específico y la mortalidad por cáncer de próstata. CCM. 2017; 21(1): 100-11.

\section{Correspondencia:}

Nancy Victoria Castilla Torres

Dirección: Jr. Ángel del Señor Mz “C” lote 1-a. Ayacucho, Perú.

Teléfono: +51999626359

Correo electrónico: nancy.castilla@unsch.edu.pe

Recibido: 24 de febrero de 2021 Evaluado: 10 de marzo de 2021 Aprobado: 22 de marzo de 2021

(c) La revista. Publicado por Universidad de San Martín de Porres, Perú. (c) Br Licencia de Creative Commons Artículo en acceso abierto bajo términos de Licencia Creative Commons Atribución 4.0 Internacional. (http://creativecommons.org/licenses/by/4.0/)

ORCID iDs

Cinthia Gavilán Zamora Emilio Germán Ramírez Roca Nancy Victoria Castilla-Torres
(1) https: / / orcid.org/0000-0002-9256-6049
(1) https: / / orcid.org/0000-0002-9704-1954
() https: / / orcid.org/0000-0001-7289-0805 